Acta Crystallographica Section E

Structure Reports

Online

ISSN 1600-5368

\section{6-Bromo-2-(diprop-2-ynylamino)-1H- benzo[de]isoquinoline-1,3(2H)-dione}

\section{Jing-Song Lv, ${ }^{a, b}$ Jun-Lei Wang ${ }^{b}$ and Cheng-He Zhou ${ }^{a *}$}

aLaboratory of Bioorganic \& Medicinal Chemistry, School of Chemistry and Chemical Engineering, Southwest University, Chongqing 400715, People's Republic of China, and ${ }^{\mathbf{b}}$ School of Chemistry and Chemical Engineering, Bijie University, Bijie, Guizhou 551700, People's Republic of China

Correspondence e-mail: zhouch@swu.edu.cn

Received 23 April 2012; accepted 17 May 2012

Key indicators: single-crystal X-ray study; $T=296 \mathrm{~K}$; mean $\sigma(\mathrm{C}-\mathrm{C})=0.009 \AA$; disorder in main residue; $R$ factor $=0.067 ; w R$ factor $=0.189$; data-to-parameter ratio $=13.9$.

The asymmetric unit of the title compound, $\mathrm{C}_{18} \mathrm{H}_{11} \mathrm{BrN}_{2} \mathrm{O}_{2}$, contains two independent molecules in which the prop-2ynylamino groups have different mutual orientations. In one molecule, the $\mathrm{Br}$ atom is disordered over two positions, with refined occupancies of 0.742 (2) and 0.258 (2).

\section{Related literature}

For the applications and biological activity of naphthalimide compounds, see: Muth et al. (2007); Zhang \& Zhou (2011); Zhang et al. (2011). For the synthesis, see: Wang et al. (2010).

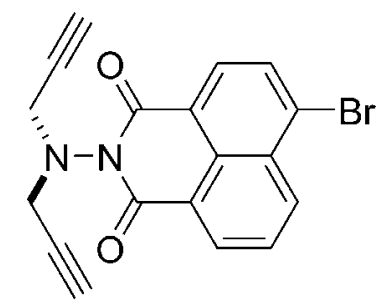

\section{Experimental}

\section{Crystal data}

$\mathrm{C}_{18} \mathrm{H}_{11} \mathrm{BrN}_{2} \mathrm{O}_{2}$

$M_{r}=367.20$

Triclinic, $P \overline{1}$

$$
\begin{aligned}
& \alpha=75.128(4)^{\circ} \\
& \beta=75.725(4)^{\circ} \\
& \gamma=72.298(4)^{\circ} \\
& V=1517.2(7) \AA^{3} \\
& Z=4
\end{aligned}
$$

Data collection

Bruker APEXII CCD diffractometer

Absorption correction: multi-scan (SADABS; Sheldrick, 1996)

$T_{\text {min }}=0.538, T_{\text {max }}=0.640$

\section{Refinement}

$R\left[F^{2}>2 \sigma\left(F^{2}\right)\right]=0.067$

$w R\left(F^{2}\right)=0.189$

$S=1.04$

5901 reflections

425 parameters
Mo $K \alpha$ radiation

$\mu=2.72 \mathrm{~mm}^{-1}$

$T=296 \mathrm{~K}$

$0.26 \times 0.24 \times 0.18 \mathrm{~mm}$

8441 measured reflections 5901 independent reflections 3709 reflections with $I>2 \sigma(I)$ $R_{\text {int }}=0.023$

Data collection: APEX2 (Bruker, 2001); cell refinement: SAINT (Bruker, 2001); data reduction: $S A I N T$; program(s) used to solve structure: SHELXS97 (Sheldrick, 2008); program(s) used to refine structure: SHELXL97 (Sheldrick, 2008); molecular graphics: PLATON (Spek, 2009); software used to prepare material for publication: SHELXTL (Sheldrick, 2008).

This work was partially supported by the National Natural Science Foundation of China (No. 21172181), the key program of the Natural Science Foundation of Chongqing (CSTC2012jjB10026), the Specialized Research Fund for the Doctoral Program of Higher Education of China (SRFDP 20110182110007), the Research Funds for the Central Universities (XDJK2012B026) and the Early Career Development Fellowship Program of Bijie University (20102002).

Supplementary data and figures for this paper are available from the IUCr electronic archives (Reference: LH5464).

\title{
References
}

Bruker (2001). APEX2 and SAINT. Bruker AXS Inc., Madison, Wisconsin, USA.

Muth, M., Hoerr, V., Glaser, M., Ponte-Sucre, A., Moll, H., Stich, A. \& Holzgrabe, U. (2007). Bioorg. Med. Chem. Lett. 17, 1590-1593.

Sheldrick, G. M. (1996). SADABS. University of Göttingen, Germany.

Sheldrick, G. M. (2008). Acta Cryst. A64, 112-122.

Spek, A. L. (2009). Acta Cryst. D65, 148-155.

Wang, X.-L., Wan, K. \& Zhou, C.-H. (2010). Eur. J. Med. Chem. 45, 5631-4639.

Zhang, Y.-Y., Mi, J.-L., Zhou, C.-H. \& Zhou, X.-D. (2011). Eur. J. Med. Chem. 46, 4391-4402.

Zhang, Y.-Y. \& Zhou, C.-H. (2011). Bioorg. Med. Chem. Lett. 21, 4349-4352. 


\section{supporting information}

Acta Cryst. (2012). E68, o1852 [doi:10.1107/S1600536812022507]

\section{6-Bromo-2-(diprop-2-ynylamino)-1 H-benzo[de]isoquinoline-1,3(2H)-dione}

\section{Jing-Song Lv, Jun-Lei Wang and Cheng-He Zhou}

\section{S1. Comment}

Naphthalimide compounds are an important type of cyclic imides with strong hydrophobicity and a desirable large $\pi$ conjugated backbone. This special aromatic heterocycle can easily interact with various active targets in biological systems via non-covalent forces such as $\pi \cdots \pi$ stacking (Muth et al., 2007), and exhibit diverse biological activities including anticancer, antibacterial, antitrypanosomal, analgesic, photobiological and antinociceptive potency (Zhang \& Zhou, 2011). Our interest is to develop novel naphthalimide-derived compounds as potential antimicrobial agents (Zhang et al., 2011). Herein, the crystal structure of the title compound (I) is reported.

The asymmetric unit of (I) is shown in Fig. 1. In the two independent molecules, the prop-2-ynylamino groups have different mutual orientations (see Fig. 1). In one moeclue the $\mathrm{Br}$ atom is disordered over two positions with refined occupancies $0.742(2)$ and $0.258(2)$.

\section{S2. Experimental}

Compound (I) was prepared according to the procedure of Wang et al. (2010). A mixture of 2-amino-6-bromo-1Hbenzo[de]isoquinoline-1,3(2H)-dione $(1.00 \mathrm{~g}, 3.44 \mathrm{mmol})$, anhydrous potassium carbonate $(1.43 \mathrm{~g}, 10.31 \mathrm{mmol})$ and propargyl bromide $(1.02 \mathrm{~g}, 8.59 \mathrm{mmol})$ in $N, N$-dimethylacetamide $(20 \mathrm{ml})$ was stirred at $353 \mathrm{~K}$. After the reaction was complete (monitored by TLC, petroleum ether/ethyl acetate, $3 / 1, \mathrm{~V} / \mathrm{V}$ ), the solvent was removed. The residue was dissolved in dichloromethane $(30 \mathrm{ml})$ and washed with water $(3 \times 30 \mathrm{ml})$. The organic phase was dried over anhydrous sodium sulfate, concentrated under reduced pressure and then purified by silica gel column chromatography eluting with petroleum ether/ethyl acetate (10/1-4/1, V/V) to give (I) as yellow solid. Crystals suitable for X-ray analysis were grown from a mixed solution of (I) in ethyl acetate and petroleum ether by slow evaporation at room temperature.

\section{S3. Refinement}

$\mathrm{H}$ atoms were positioned geometrically and refined using a riding model, with $\mathrm{C}-\mathrm{H}=0.93-0.97 \AA$ and with $U_{\text {iso }}(\mathrm{H})=$ $1.2 U_{\mathrm{eq}}(\mathrm{C})$. 


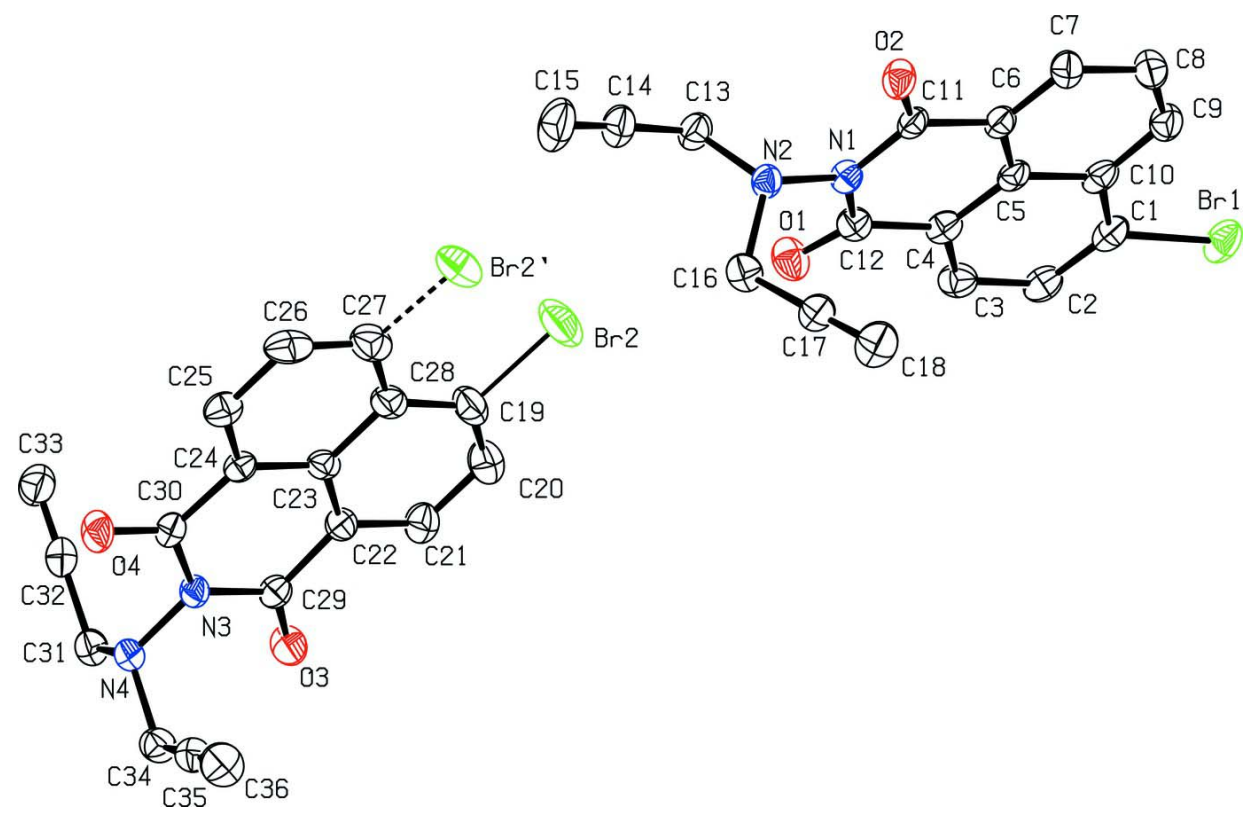

\section{Figure 1}

The asymmetric unit of (I), showing displacement ellipsoids are drawn at the $30 \%$ probability level. The minor component of disorder is shown with a dashed bond.

\section{6-Bromo-2-(diprop-2-ynylamino)-1 $H$-benzo[de]isoquinoline- 1,3(2H)-dione}

Crystal data

$\mathrm{C}_{18} \mathrm{H}_{11} \mathrm{BrN}_{2} \mathrm{O}_{2}$

$M_{r}=367.20$

Triclinic, $P \overline{1}$

Hall symbol: -P 1

$a=10.460(3) \AA$

$b=11.816(3) \AA$

$c=13.561(3) \AA$

$\alpha=75.128(4)^{\circ}$

$\beta=75.725(4)^{\circ}$

$\gamma=72.298(4)^{\circ}$

$V=1517.2(7) \AA^{3}$

Data collection

Bruker APEXII CCD

diffractometer

Radiation source: fine-focus sealed tube

Graphite monochromator

$\varphi$ and $\omega$ scans

Absorption correction: multi-scan

(SADABS; Sheldrick, 1996)

$T_{\min }=0.538, T_{\max }=0.640$
$Z=4$

$F(000)=736$

$D_{\mathrm{x}}=1.608 \mathrm{Mg} \mathrm{m}^{-3}$

Mo $K \alpha$ radiation, $\lambda=0.71073 \AA$

Cell parameters from 1880 reflections

$\theta=2.4-23.5^{\circ}$

$\mu=2.72 \mathrm{~mm}^{-1}$

$T=296 \mathrm{~K}$

Block, yellow

$0.26 \times 0.24 \times 0.18 \mathrm{~mm}$

8441 measured reflections

5901 independent reflections

3709 reflections with $I>2 \sigma(I)$

$R_{\text {int }}=0.023$

$\theta_{\text {max }}=26.0^{\circ}, \theta_{\min }=1.6^{\circ}$

$h=-8 \rightarrow 12$

$k=-12 \rightarrow 14$

$l=-14 \rightarrow 16$ 


\section{Refinement}

Refinement on $F^{2}$

Least-squares matrix: full

$R\left[F^{2}>2 \sigma\left(F^{2}\right)\right]=0.067$

$w R\left(F^{2}\right)=0.189$

$S=1.04$

5901 reflections

425 parameters

1 restraint

Primary atom site location: structure-invariant direct methods
Secondary atom site location: difference Fourier map

Hydrogen site location: inferred from neighbouring sites

$\mathrm{H}$-atom parameters constrained

$w=1 /\left[\sigma^{2}\left(F_{\mathrm{o}}^{2}\right)+(0.0813 P)^{2}+2.8703 P\right]$ where $P=\left(F_{\mathrm{o}}{ }^{2}+2 F_{\mathrm{c}}{ }^{2}\right) / 3$

$(\Delta / \sigma)_{\max }<0.001$

$\Delta \rho_{\max }=2.20 \mathrm{e}^{-3}$

$\Delta \rho_{\min }=-0.74$ e $\AA^{-3}$

Special details

Geometry. All e.s.d.'s (except the e.s.d. in the dihedral angle between two l.s. planes) are estimated using the full covariance matrix. The cell e.s.d.'s are taken into account individually in the estimation of e.s.d.'s in distances, angles and torsion angles; correlations between e.s.d.'s in cell parameters are only used when they are defined by crystal symmetry. An approximate (isotropic) treatment of cell e.s.d.'s is used for estimating e.s.d.'s involving 1.s. planes.

Refinement. Refinement of $F^{2}$ against ALL reflections. The weighted $R$-factor $w R$ and goodness of fit $S$ are based on $F^{2}$, conventional $R$-factors $R$ are based on $F$, with $F$ set to zero for negative $F^{2}$. The threshold expression of $F^{2}>\sigma\left(F^{2}\right)$ is used only for calculating $R$-factors (gt) etc. and is not relevant to the choice of reflections for refinement. $R$-factors based on $F^{2}$ are statistically about twice as large as those based on $F$, and $R$ - factors based on ALL data will be even larger.

Fractional atomic coordinates and isotropic or equivalent isotropic displacement parameters $\left(\AA^{2}\right)$

\begin{tabular}{|c|c|c|c|c|c|}
\hline & $x$ & $y$ & $z$ & $U_{\text {iso }} * / U_{\text {eq }}$ & Occ. $(<1)$ \\
\hline $\mathrm{Br} 2$ & $0.55459(14)$ & $0.50977(11)$ & $0.76724(11)$ & $0.0989(5)$ & $0.742(2)$ \\
\hline C19 & $0.6750(6)$ & $0.5968(6)$ & $0.7825(5)$ & $0.0631(18)$ & \\
\hline $\mathrm{C} 27$ & $0.4943(7)$ & $0.6809(6)$ & $0.9230(5)$ & $0.0630(18)$ & \\
\hline $\mathrm{H} 27$ & 0.4352 & 0.6387 & 0.9177 & $0.076^{*}$ & $0.742(2)$ \\
\hline $\mathrm{Br} 2^{\prime}$ & $0.3819(3)$ & 0.5988 & $0.9048(3)$ & $0.0711(11)$ & $0.258(2)$ \\
\hline H19' & 0.6195 & 0.5535 & 0.7732 & $0.076^{*}$ & $0.258(2)$ \\
\hline $\mathrm{O} 3$ & $1.0432(4)$ & $0.7900(4)$ & 0.7554 & $0.0576(11)$ & \\
\hline $\mathrm{O} 4$ & $0.7320(4)$ & $0.9391(4)$ & $1.0192(3)$ & $0.0579(11)$ & \\
\hline N3 & $0.8868(4)$ & $0.8680(4)$ & 0.8839 & $0.0391(10)$ & \\
\hline N4 & $0.9653(5)$ & $0.9410(4)$ & 0.8921 & $0.0429(11)$ & \\
\hline $\mathrm{C} 20$ & $0.7978(8)$ & $0.5865(6)$ & $0.7231(5)$ & $0.0658(18)$ & \\
\hline $\mathrm{H} 20 \mathrm{~A}$ & 0.8266 & 0.5359 & 0.6751 & $0.079 *$ & \\
\hline $\mathrm{C} 21$ & $0.8830(7)$ & $0.6511(6)$ & $0.7325(5)$ & $0.0554(16)$ & \\
\hline $\mathrm{H} 21 \mathrm{~A}$ & 0.9684 & 0.6451 & 0.6899 & $0.067 *$ & \\
\hline $\mathrm{C} 22$ & $0.8417(6)$ & $0.7239(5)$ & $0.8044(4)$ & $0.0440(13)$ & \\
\hline $\mathrm{C} 23$ & $0.7107(5)$ & $0.7359(5)$ & 0.8683 & $0.0398(12)$ & \\
\hline $\mathrm{C} 24$ & $0.6694(5)$ & $0.8110(5)$ & $0.9422(4)$ & $0.0384(12)$ & \\
\hline $\mathrm{C} 25$ & $0.5441(6)$ & $0.8219(6)$ & $1.0036(4)$ & $0.0512(15)$ & \\
\hline $\mathrm{H} 25 \mathrm{~A}$ & 0.5160 & 0.8725 & 1.0516 & $0.061 *$ & \\
\hline $\mathrm{C} 26$ & $0.4565(6)$ & $0.7562(6)$ & $0.9943(5)$ & $0.0622(18)$ & \\
\hline $\mathrm{H} 26 \mathrm{~A}$ & 0.3711 & 0.7637 & 1.0372 & $0.075 *$ & \\
\hline $\mathrm{C} 28$ & $0.6227(6)$ & $0.6695(5)$ & $0.8594(5)$ & $0.0506(15)$ & \\
\hline $\mathrm{C} 29$ & $0.9338(6)$ & $0.7938(5)$ & $0.8102(4)$ & $0.0415(13)$ & \\
\hline $\mathrm{C} 30$ & $0.7610(5)$ & $0.8777(5)$ & $0.9544(4)$ & $0.0416(13)$ & \\
\hline $\mathrm{C} 31$ & $1.0955(6)$ & $0.8715(6)$ & $0.9279(5)$ & $0.0524(15)$ & \\
\hline
\end{tabular}




\begin{tabular}{|c|c|c|c|c|}
\hline H31A & 1.1567 & 0.8304 & 0.8741 & $0.063^{*}$ \\
\hline H31B & 1.1389 & 0.9269 & 0.9406 & $0.063 *$ \\
\hline $\mathrm{C} 32$ & $1.0719(6)$ & $0.7828(6)$ & $1.0224(5)$ & $0.0516(15)$ \\
\hline $\mathrm{C} 33$ & $1.0549(7)$ & $0.7110(6)$ & $1.0983(5)$ & $0.0650(18)$ \\
\hline $\mathrm{H} 33$ & 1.0414 & 0.6537 & 1.1587 & $0.078 *$ \\
\hline $\mathrm{C} 34$ & $0.9868(6)$ & $1.0320(5)$ & $0.7990(5)$ & $0.0529(15)$ \\
\hline H34A & 1.0319 & 1.0855 & 0.8126 & $0.063 *$ \\
\hline H34B & 1.0468 & 0.9922 & 0.7440 & $0.063^{*}$ \\
\hline $\mathrm{C} 35$ & $0.8608(7)$ & $1.1035(6)$ & $0.7643(5)$ & $0.0513(15)$ \\
\hline $\mathrm{C} 36$ & $0.7616(8)$ & $1.1615(7)$ & $0.7340(6)$ & $0.073(2)$ \\
\hline H36 & 0.6821 & 1.2079 & 0.7098 & $0.088^{*}$ \\
\hline $\mathrm{Br} 1$ & $0.59705(8)$ & $0.00106(6)$ & $0.23760(5)$ & $0.0651(3)$ \\
\hline $\mathrm{O} 1$ & 0.5025 & $0.3271(4)$ & $0.6119(4)$ & $0.0619(12)$ \\
\hline $\mathrm{O} 2$ & $0.0699(4)$ & 0.2945 (4) & $0.6614(3)$ & $0.0534(10)$ \\
\hline $\mathrm{N} 1$ & 0.2845 & 0.3213 (4) & $0.6295(3)$ & $0.0384(10)$ \\
\hline $\mathrm{N} 2$ & $0.2348(5)$ & $0.4032(4)$ & $0.6985(3)$ & $0.0411(10)$ \\
\hline $\mathrm{C} 1$ & $0.5383(7)$ & $0.0905(5)$ & $0.3449(4)$ & $0.0507(15)$ \\
\hline $\mathrm{C} 2$ & $0.6289(6)$ & $0.1396(6)$ & $0.3650(4)$ & $0.0513(15)$ \\
\hline $\mathrm{H} 2 \mathrm{~A}$ & 0.7169 & 0.1289 & 0.3260 & $0.062 *$ \\
\hline $\mathrm{C} 3$ & $0.5900(6)$ & $0.2068(5)$ & $0.4446(5)$ & $0.0526(15)$ \\
\hline $\mathrm{H} 3 \mathrm{~A}$ & 0.6525 & 0.2389 & 0.4593 & $0.063^{*}$ \\
\hline $\mathrm{C} 4$ & $0.4603(6)$ & $0.2243(5)$ & $0.4999(4)$ & $0.0431(13)$ \\
\hline $\mathrm{C} 5$ & $0.3643(6)$ & 0.1758 (4) & $0.4791(4)$ & $0.0412(13)$ \\
\hline C6 & $0.2311(6)$ & $0.1948(5)$ & $0.5347(4)$ & $0.0417(13)$ \\
\hline $\mathrm{C} 7$ & $0.1384(6)$ & $0.1445(5)$ & $0.5174(4)$ & $0.0500(14)$ \\
\hline H7A & 0.0501 & 0.1577 & 0.5557 & $0.060 *$ \\
\hline $\mathrm{C} 8$ & $0.1779(7)$ & $0.0727(6)$ & $0.4415(5)$ & $0.0566(16)$ \\
\hline H8A & 0.1162 & 0.0370 & 0.4304 & $0.068 *$ \\
\hline C9 & $0.3051(7)$ & $0.0557(5)$ & $0.3849(4)$ & $0.0536(15)$ \\
\hline H9A & 0.3286 & 0.0097 & 0.3338 & $0.064 *$ \\
\hline $\mathrm{C} 10$ & $0.4030(6)$ & $0.1046(5)$ & $0.4001(4)$ & $0.0470(14)$ \\
\hline $\mathrm{C} 11$ & $0.1848(6)$ & $0.2737(5)$ & $0.6133(4)$ & $0.0405(13)$ \\
\hline $\mathrm{C} 12$ & $0.4232(6)$ & $0.2937(5)$ & $0.5833(4)$ & $0.0460(13)$ \\
\hline $\mathrm{C} 13$ & $0.2805(6)$ & $0.3486(5)$ & $0.7979(4)$ & $0.0514(15)$ \\
\hline H13A & 0.2603 & 0.2703 & 0.8244 & $0.062 *$ \\
\hline H13B & 0.3784 & 0.3363 & 0.7882 & $0.062 *$ \\
\hline $\mathrm{C} 14$ & $0.2116(7)$ & $0.4275(6)$ & $0.8720(5)$ & $0.0590(16)$ \\
\hline $\mathrm{C} 15$ & $0.1620(9)$ & $0.4915(8)$ & $0.9295(6)$ & $0.091(3)$ \\
\hline H15 & 0.1218 & 0.5435 & 0.9762 & $0.109^{*}$ \\
\hline $\mathrm{C} 16$ & $0.2598(6)$ & $0.5219(5)$ & $0.6495(5)$ & $0.0499(14)$ \\
\hline H16A & 0.2196 & 0.5768 & 0.6977 & $0.060 *$ \\
\hline H16B & 0.3573 & 0.5144 & 0.6322 & $0.060 *$ \\
\hline $\mathrm{C} 17$ & $0.2019(6)$ & $0.5705(5)$ & $0.5570(5)$ & $0.0508(15)$ \\
\hline $\mathrm{C} 18$ & $0.1566(8)$ & $0.6073(6)$ & $0.4818(6)$ & $0.073(2)$ \\
\hline H18 & 0.1203 & 0.6370 & 0.4213 & $0.088^{*}$ \\
\hline
\end{tabular}


Atomic displacement parameters $\left(\AA^{2}\right)$

\begin{tabular}{|c|c|c|c|c|c|c|}
\hline & $U^{11}$ & $U^{22}$ & $U^{33}$ & $U^{12}$ & $U^{13}$ & $U^{23}$ \\
\hline $\mathrm{Br} 2$ & $0.1274(11)$ & $0.0833(8)$ & $0.1227(10)$ & $-0.0587(8)$ & $-0.0590(8)$ & $-0.0111(7)$ \\
\hline C19 & $0.090(5)$ & $0.042(4)$ & $0.072(4)$ & -0.024 & $-0.040(4)$ & -0.004 \\
\hline $\mathrm{C} 27$ & $0.062(4)$ & $0.057(4)$ & $0.073(4)$ & -0.021 & $-0.025(4)$ & $0.000(4)$ \\
\hline $\mathrm{Br} 2^{\prime}$ & $0.0594(18)$ & $0.0600(18)$ & $0.103(2)$ & $-0.0243(13)$ & $-0.0217(15)$ & $-0.0145(15)$ \\
\hline C19' & $0.090(5)$ & $0.042(4)$ & $0.072(4)$ & -0.024 & $-0.040(4)$ & -0.004 \\
\hline $\mathrm{C} 27^{\prime}$ & $0.062(4)$ & $0.057(4)$ & $0.073(4)$ & -0.021 & $-0.025(4)$ & $0.000(4)$ \\
\hline $\mathrm{O} 3$ & $0.045(2)$ & $0.071(3)$ & $0.062(3)$ & $-0.021(2)$ & $0.011(2)$ & $-0.034(2)$ \\
\hline $\mathrm{O} 4$ & $0.056(3)$ & $0.072(3)$ & $0.053(2)$ & $-0.019(2)$ & $0.008(2)$ & $-0.038(2)$ \\
\hline N3 & $0.040(3)$ & $0.043(3)$ & 0.037 (2) & $-0.012(2)$ & $-0.0010(19)$ & $-0.0156(19)$ \\
\hline N4 & $0.046(3)$ & $0.046(3)$ & $0.041(2)$ & $-0.019(2)$ & $-0.008(2)$ & $-0.010(2)$ \\
\hline C20 & $0.075(5)$ & $0.059(4)$ & $0.072(4)$ & $-0.016(4)$ & $-0.016(4)$ & $-0.026(4)$ \\
\hline $\mathrm{C} 21$ & $0.060(4)$ & $0.056(4)$ & $0.051(3)$ & -0.009 (3) & -0.010 & $-0.018(3)$ \\
\hline $\mathrm{C} 22$ & $0.054(4)$ & $0.035(3)$ & $0.042(3)$ & -0.010 & $-0.007(3)$ & $-0.010(2)$ \\
\hline $\mathrm{C} 23$ & $0.041(3)$ & $0.036(3)$ & $0.042(3)$ & $-0.012(2)$ & $-0.015(2)$ & $0.002(2)$ \\
\hline $\mathrm{C} 24$ & $0.040(3)$ & $0.037(3)$ & $0.035(3)$ & $-0.007(2)$ & $-0.007(2)$ & $-0.003(2)$ \\
\hline $\mathrm{C} 25$ & $0.044(4)$ & $0.055(4)$ & $0.048(3)$ & $-0.011(3)$ & $-0.009(3)$ & $-0.002(3)$ \\
\hline $\mathrm{C} 26$ & $0.033(3)$ & $0.071(5)$ & $0.071(4)$ & $-0.019(3)$ & $-0.009(3)$ & $0.011(4)$ \\
\hline $\mathrm{C} 28$ & $0.058(4)$ & $0.042(3)$ & $0.055(3)$ & $-0.016(3)$ & $-0.024(3)$ & $0.003(3)$ \\
\hline C29 & $0.040(3)$ & $0.043(3)$ & $0.039(3)$ & $-0.008(2)$ & -0.005 & -0.009 (2) \\
\hline C30 & $0.045(3)$ & $0.044(3)$ & $0.035(3)$ & $-0.007(2)$ & $-0.008(2)$ & $-0.010(2)$ \\
\hline C31 & $0.055(4)$ & $0.059(4)$ & $0.050(3)$ & $-0.022(3)$ & $-0.011(3)$ & $-0.014(3)$ \\
\hline C32 & $0.055(4)$ & $0.057(4)$ & $0.048(3)$ & $-0.013(3)$ & $-0.012(3)$ & -0.019 \\
\hline C33 & $0.080(5)$ & $0.064(4)$ & $0.051(4)$ & $-0.017(4)$ & $-0.011(3)$ & $-0.016(3)$ \\
\hline C34 & $0.058(4)$ & $0.048(4)$ & $0.054(3)$ & $-0.018(3)$ & $-0.002(3)$ & $-0.014(3)$ \\
\hline C35 & $0.056(4)$ & $0.049(4)$ & $0.050(3)$ & $-0.017(3)$ & $-0.012(3)$ & $-0.006(3)$ \\
\hline C36 & $0.072(5)$ & $0.067(5)$ & $0.081(5)$ & $-0.014(4)$ & $-0.024(4)$ & $-0.011(4)$ \\
\hline $\mathrm{Br} 1$ & $0.0852(5)$ & $0.0556(4)$ & 0.0410 & -0.0003 & -0.0023 & $-0.0167(3)$ \\
\hline $\mathrm{O} 1$ & 0.047 (3) & $0.075(3)$ & $0.073(3)$ & $-0.019(2)$ & $-0.005(2)$ & $-0.031(2)$ \\
\hline $\mathrm{O} 2$ & $0.044(2)$ & $0.075(3)$ & $0.046(2)$ & $-0.018(2)$ & $-0.0006(19)$ & $-0.024(2)$ \\
\hline N1 & $0.035(2)$ & $0.038(2)$ & $0.038(2)$ & $-0.0026(19)$ & -0.0018 (19) & $-0.0125(19)$ \\
\hline N2 & $0.050(3)$ & $0.036(2)$ & $0.038(2)$ & $-0.007(2)$ & $-0.011(2)$ & -0.0098 (19) \\
\hline $\mathrm{C} 1$ & $0.063(4)$ & $0.041(3)$ & $0.034(3)$ & $-0.001(3)$ & $-0.004(3)$ & -0.005 (2) \\
\hline $\mathrm{C} 2$ & $0.041(3)$ & $0.060(4)$ & $0.042(3)$ & -0.007 & $0.006(3)$ & $-0.011(3)$ \\
\hline $\mathrm{C} 3$ & $0.053(4)$ & $0.045(3)$ & $0.054(3)$ & -0.011 & -0.003 & -0.008 \\
\hline $\mathrm{C} 4$ & $0.043(3)$ & $0.037(3)$ & $0.039(3)$ & $-0.001(2)$ & $-0.004(2)$ & $-0.004(2)$ \\
\hline $\mathrm{C} 5$ & $0.056(4)$ & $0.030(3)$ & $0.030(2)$ & $-0.004(2)$ & $-0.011(2)$ & $0.001(2)$ \\
\hline C6 & $0.050(3)$ & $0.039(3)$ & $0.032(3)$ & $-0.008(2)$ & $-0.008(2)$ & $-0.005(2)$ \\
\hline $\mathrm{C} 7$ & $0.055(4)$ & $0.051(4)$ & $0.046(3)$ & $-0.012(3)$ & $-0.011(3)$ & $-0.015(3)$ \\
\hline $\mathrm{C} 8$ & $0.066(4)$ & $0.057(4)$ & $0.056(4)$ & $-0.020(3)$ & $-0.018(3)$ & -0.015 \\
\hline C9 & $0.071(5)$ & $0.046(4)$ & $0.044(3)$ & $-0.012(3)$ & $-0.011(3)$ & $-0.013(3)$ \\
\hline $\mathrm{C} 10$ & $0.058(4)$ & $0.037(3)$ & $0.033(3)$ & $-0.002(3)$ & $-0.007(3)$ & $0.001(2)$ \\
\hline C11 & $0.050(4)$ & $0.040(3)$ & $0.029(2)$ & -0.009 (3) & -0.009 (3) & $-0.003(2)$ \\
\hline C12 & $0.046(4)$ & $0.041(3)$ & $0.048(3)$ & $-0.008(3)$ & $-0.012(3)$ & $-0.006(3)$ \\
\hline C13 & $0.061(4)$ & $0.052(4)$ & $0.040(3)$ & -0.010 & -0.015 & $-0.006(3)$ \\
\hline C14 & $0.069(4)$ & $0.061(4)$ & $0.048(3)$ & -0.010 & $-0.012(3)$ & -0.020 \\
\hline
\end{tabular}




\begin{tabular}{lllllll}
$\mathrm{C} 15$ & $0.131(7)$ & $0.081(6)$ & $0.060(4)$ & $-0.008(5)$ & $-0.021(5)$ & $-0.032(4)$ \\
$\mathrm{C} 16$ & $0.050(3)$ & $0.040(3)$ & $0.059(3)$ & $-0.003(3)$ & $-0.018(3)$ & $-0.010(3)$ \\
$\mathrm{C} 17$ & $0.061(4)$ & $0.038(3)$ & $0.047(3)$ & $-0.002(3)$ & $-0.010(3)$ & $-0.009(3)$ \\
$\mathrm{C} 18$ & $0.089(5)$ & $0.054(4)$ & $0.066(4)$ & $0.002(4)$ & $-0.020(4)$ & $-0.012(3)$ \\
\hline
\end{tabular}

Geometric parameters $\left(A,{ }^{\circ}\right)$

\begin{tabular}{|c|c|c|c|}
\hline $\mathrm{Br} 2-\mathrm{C} 19$ & $1.927(6)$ & $\mathrm{Br} 1-\mathrm{C} 1$ & $1.888(6)$ \\
\hline $\mathrm{C} 19-\mathrm{C} 20$ & $1.326(9)$ & $\mathrm{O} 1-\mathrm{C} 12$ & $1.197(7)$ \\
\hline $\mathrm{C} 19-\mathrm{C} 28$ & $1.421(9)$ & $\mathrm{O} 2-\mathrm{C} 11$ & $1.202(6)$ \\
\hline $\mathrm{C} 27-\mathrm{C} 26$ & $1.386(9)$ & $\mathrm{N} 1-\mathrm{C} 12$ & $1.405(7)$ \\
\hline $\mathrm{C} 27-\mathrm{C} 28$ & $1.394(9)$ & $\mathrm{N} 1-\mathrm{C} 11$ & $1.409(7)$ \\
\hline $\mathrm{C} 27-\mathrm{H} 27$ & 0.9300 & $\mathrm{~N} 1-\mathrm{N} 2$ & $1.415(6)$ \\
\hline $\mathrm{O} 3-\mathrm{C} 29$ & $1.196(6)$ & $\mathrm{N} 2-\mathrm{C} 16$ & $1.462(7)$ \\
\hline $\mathrm{O} 4-\mathrm{C} 30$ & $1.205(6)$ & $\mathrm{N} 2-\mathrm{C} 13$ & $1.465(7)$ \\
\hline $\mathrm{N} 3-\mathrm{C} 29$ & $1.397(7)$ & $\mathrm{C} 1-\mathrm{C} 2$ & $1.361(9)$ \\
\hline $\mathrm{N} 3-\mathrm{N} 4$ & $1.399(6)$ & $\mathrm{C} 1-\mathrm{C} 10$ & $1.411(8)$ \\
\hline $\mathrm{N} 3-\mathrm{C} 30$ & $1.416(7)$ & $\mathrm{C} 2-\mathrm{C} 3$ & $1.410(8)$ \\
\hline $\mathrm{N} 4-\mathrm{C} 34$ & $1.452(7)$ & $\mathrm{C} 2-\mathrm{H} 2 \mathrm{~A}$ & 0.9300 \\
\hline $\mathrm{N} 4-\mathrm{C} 31$ & $1.486(7)$ & $\mathrm{C} 3-\mathrm{C} 4$ & $1.361(8)$ \\
\hline $\mathrm{C} 20-\mathrm{C} 21$ & $1.383(9)$ & $\mathrm{C} 3-\mathrm{H} 3 \mathrm{~A}$ & 0.9300 \\
\hline $\mathrm{C} 20-\mathrm{H} 20 \mathrm{~A}$ & 0.9300 & $\mathrm{C} 4-\mathrm{C} 5$ & $1.409(8)$ \\
\hline $\mathrm{C} 21-\mathrm{C} 22$ & $1.369(8)$ & $\mathrm{C} 4-\mathrm{C} 12$ & $1.473(8)$ \\
\hline $\mathrm{C} 21-\mathrm{H} 21 \mathrm{~A}$ & 0.9300 & $\mathrm{C} 5-\mathrm{C} 6$ & $1.392(8)$ \\
\hline $\mathrm{C} 22-\mathrm{C} 23$ & $1.417(8)$ & $\mathrm{C} 5-\mathrm{C} 10$ & $1.437(8)$ \\
\hline $\mathrm{C} 22-\mathrm{C} 29$ & $1.474(8)$ & $\mathrm{C} 6-\mathrm{C} 7$ & $1.372(8)$ \\
\hline $\mathrm{C} 23-\mathrm{C} 24$ & $1.411(7)$ & $\mathrm{C} 6-\mathrm{C} 11$ & $1.491(7)$ \\
\hline $\mathrm{C} 23-\mathrm{C} 28$ & $1.421(8)$ & $\mathrm{C} 7-\mathrm{C} 8$ & $1.405(8)$ \\
\hline $\mathrm{C} 24-\mathrm{C} 25$ & $1.357(8)$ & $\mathrm{C} 7-\mathrm{H} 7 \mathrm{~A}$ & 0.9300 \\
\hline $\mathrm{C} 24-\mathrm{C} 30$ & $1.473(8)$ & $\mathrm{C} 8-\mathrm{C} 9$ & $1.346(9)$ \\
\hline $\mathrm{C} 25-\mathrm{C} 26$ & $1.414(9)$ & $\mathrm{C} 8-\mathrm{H} 8 \mathrm{~A}$ & 0.9300 \\
\hline $\mathrm{C} 25-\mathrm{H} 25 \mathrm{~A}$ & 0.9300 & $\mathrm{C} 9-\mathrm{C} 10$ & $1.396(9)$ \\
\hline $\mathrm{C} 26-\mathrm{H} 26 \mathrm{~A}$ & 0.9300 & C9-H9A & 0.9300 \\
\hline $\mathrm{C} 31-\mathrm{C} 32$ & $1.454(9)$ & $\mathrm{C} 13-\mathrm{C} 14$ & $1.453(8)$ \\
\hline $\mathrm{C} 31-\mathrm{H} 31 \mathrm{~A}$ & 0.9700 & $\mathrm{C} 13-\mathrm{H} 13 \mathrm{~A}$ & 0.9700 \\
\hline $\mathrm{C} 31-\mathrm{H} 31 \mathrm{~B}$ & 0.9700 & C13-H13B & 0.9700 \\
\hline $\mathrm{C} 32-\mathrm{C} 33$ & $1.168(9)$ & $\mathrm{C} 14-\mathrm{C} 15$ & $1.147(9)$ \\
\hline $\mathrm{C} 33-\mathrm{H} 33$ & 0.9300 & C15-H15 & 0.9300 \\
\hline $\mathrm{C} 34-\mathrm{C} 35$ & $1.449(9)$ & $\mathrm{C} 16-\mathrm{C} 17$ & $1.436(8)$ \\
\hline $\mathrm{C} 34-\mathrm{H} 34 \mathrm{~A}$ & 0.9700 & C16-H16A & 0.9700 \\
\hline С $34-\mathrm{H} 34 \mathrm{~B}$ & 0.9700 & C16-H16B & 0.9700 \\
\hline $\mathrm{C} 35-\mathrm{C} 36$ & $1.161(9)$ & $\mathrm{C} 17-\mathrm{C} 18$ & $1.157(9)$ \\
\hline $\mathrm{C} 36-\mathrm{H} 36$ & 0.9300 & $\mathrm{C} 18-\mathrm{H} 18$ & 0.9300 \\
\hline $\mathrm{C} 20-\mathrm{C} 19-\mathrm{C} 28$ & $124.4(6)$ & $\mathrm{C} 12-\mathrm{N} 1-\mathrm{C} 11$ & $125.4(4)$ \\
\hline $\mathrm{C} 20-\mathrm{C} 19-\mathrm{Br} 2$ & $120.0(5)$ & $\mathrm{C} 12-\mathrm{N} 1-\mathrm{N} 2$ & $120.1(4)$ \\
\hline $\mathrm{C} 28-\mathrm{C} 19-\mathrm{Br} 2$ & $115.6(5)$ & $\mathrm{C} 11-\mathrm{N} 1-\mathrm{N} 2$ & $114.5(4)$ \\
\hline $\mathrm{C} 26-\mathrm{C} 27-\mathrm{C} 28$ & $118.8(6)$ & $\mathrm{N} 1-\mathrm{N} 2-\mathrm{C} 16$ & $111.9(4)$ \\
\hline
\end{tabular}




\begin{tabular}{|c|c|c|c|}
\hline $\mathrm{C} 26-\mathrm{C} 27-\mathrm{H} 27$ & 120.6 & $\mathrm{~N} 1-\mathrm{N} 2-\mathrm{C} 13$ & $111.2(4)$ \\
\hline $\mathrm{C} 28-\mathrm{C} 27-\mathrm{H} 27$ & 120.6 & $\mathrm{C} 16-\mathrm{N} 2-\mathrm{C} 13$ & $115.4(4)$ \\
\hline $\mathrm{C} 29-\mathrm{N} 3-\mathrm{N} 4$ & $120.3(4)$ & $\mathrm{C} 2-\mathrm{C} 1-\mathrm{C} 10$ & $122.8(5)$ \\
\hline $\mathrm{C} 29-\mathrm{N} 3-\mathrm{C} 30$ & $125.2(5)$ & $\mathrm{C} 2-\mathrm{C} 1-\mathrm{Br} 1$ & $118.2(4)$ \\
\hline $\mathrm{N} 4-\mathrm{N} 3-\mathrm{C} 30$ & $114.5(4)$ & $\mathrm{C} 10-\mathrm{C} 1-\mathrm{Br} 1$ & $119.0(5)$ \\
\hline $\mathrm{N} 3-\mathrm{N} 4-\mathrm{C} 34$ & $113.2(4)$ & $\mathrm{C} 1-\mathrm{C} 2-\mathrm{C} 3$ & $120.3(5)$ \\
\hline $\mathrm{N} 3-\mathrm{N} 4-\mathrm{C} 31$ & $113.7(4)$ & $\mathrm{C} 1-\mathrm{C} 2-\mathrm{H} 2 \mathrm{~A}$ & 119.9 \\
\hline $\mathrm{C} 34-\mathrm{N} 4-\mathrm{C} 31$ & $111.7(4)$ & $\mathrm{C} 3-\mathrm{C} 2-\mathrm{H} 2 \mathrm{~A}$ & 119.9 \\
\hline $\mathrm{C} 19-\mathrm{C} 20-\mathrm{C} 21$ & $119.9(6)$ & $\mathrm{C} 4-\mathrm{C} 3-\mathrm{C} 2$ & $119.5(6)$ \\
\hline $\mathrm{C} 19-\mathrm{C} 20-\mathrm{H} 20 \mathrm{~A}$ & 120.0 & $\mathrm{C} 4-\mathrm{C} 3-\mathrm{H} 3 \mathrm{~A}$ & 120.2 \\
\hline $\mathrm{C} 21-\mathrm{C} 20-\mathrm{H} 20 \mathrm{~A}$ & 120.0 & $\mathrm{C} 2-\mathrm{C} 3-\mathrm{H} 3 \mathrm{~A}$ & 120.2 \\
\hline $\mathrm{C} 22-\mathrm{C} 21-\mathrm{C} 20$ & $120.1(6)$ & $\mathrm{C} 3-\mathrm{C} 4-\mathrm{C} 5$ & $121.1(5)$ \\
\hline $\mathrm{C} 22-\mathrm{C} 21-\mathrm{H} 21 \mathrm{~A}$ & 120.0 & $\mathrm{C} 3-\mathrm{C} 4-\mathrm{C} 12$ & $118.2(6)$ \\
\hline $\mathrm{C} 20-\mathrm{C} 21-\mathrm{H} 21 \mathrm{~A}$ & 120.0 & $\mathrm{C} 5-\mathrm{C} 4-\mathrm{C} 12$ & $120.7(5)$ \\
\hline $\mathrm{C} 21-\mathrm{C} 22-\mathrm{C} 23$ & $120.6(6)$ & $\mathrm{C} 6-\mathrm{C} 5-\mathrm{C} 4$ & $121.2(5)$ \\
\hline $\mathrm{C} 21-\mathrm{C} 22-\mathrm{C} 29$ & $118.5(5)$ & $\mathrm{C} 6-\mathrm{C} 5-\mathrm{C} 10$ & $118.6(5)$ \\
\hline $\mathrm{C} 23-\mathrm{C} 22-\mathrm{C} 29$ & $120.8(5)$ & $\mathrm{C} 4-\mathrm{C} 5-\mathrm{C} 10$ & $120.2(5)$ \\
\hline $\mathrm{C} 24-\mathrm{C} 23-\mathrm{C} 22$ & $120.1(5)$ & $\mathrm{C} 7-\mathrm{C} 6-\mathrm{C} 5$ & $121.5(5)$ \\
\hline $\mathrm{C} 24-\mathrm{C} 23-\mathrm{C} 28$ & $120.2(5)$ & $\mathrm{C} 7-\mathrm{C} 6-\mathrm{C} 11$ & $118.2(5)$ \\
\hline $\mathrm{C} 22-\mathrm{C} 23-\mathrm{C} 28$ & $119.7(5)$ & $\mathrm{C} 5-\mathrm{C} 6-\mathrm{C} 11$ & $120.2(5)$ \\
\hline $\mathrm{C} 25-\mathrm{C} 24-\mathrm{C} 23$ & $119.9(5)$ & $\mathrm{C} 6-\mathrm{C} 7-\mathrm{C} 8$ & $119.5(6)$ \\
\hline $\mathrm{C} 25-\mathrm{C} 24-\mathrm{C} 30$ & $119.2(5)$ & $\mathrm{C} 6-\mathrm{C} 7-\mathrm{H} 7 \mathrm{~A}$ & 120.2 \\
\hline $\mathrm{C} 23-\mathrm{C} 24-\mathrm{C} 30$ & $120.9(5)$ & $\mathrm{C} 8-\mathrm{C} 7-\mathrm{H} 7 \mathrm{~A}$ & 120.2 \\
\hline $\mathrm{C} 24-\mathrm{C} 25-\mathrm{C} 26$ & $119.7(6)$ & $\mathrm{C} 9-\mathrm{C} 8-\mathrm{C} 7$ & $120.0(6)$ \\
\hline $\mathrm{C} 24-\mathrm{C} 25-\mathrm{H} 25 \mathrm{~A}$ & 120.1 & $\mathrm{C} 9-\mathrm{C} 8-\mathrm{H} 8 \mathrm{~A}$ & 120.0 \\
\hline $\mathrm{C} 26-\mathrm{C} 25-\mathrm{H} 25 \mathrm{~A}$ & 120.1 & $\mathrm{C} 7-\mathrm{C} 8-\mathrm{H} 8 \mathrm{~A}$ & 120.0 \\
\hline $\mathrm{C} 27-\mathrm{C} 26-\mathrm{C} 25$ & $121.8(6)$ & $\mathrm{C} 8-\mathrm{C} 9-\mathrm{C} 10$ & $122.5(6)$ \\
\hline $\mathrm{C} 27-\mathrm{C} 26-\mathrm{H} 26 \mathrm{~A}$ & 119.1 & $\mathrm{C} 8-\mathrm{C} 9-\mathrm{H} 9 \mathrm{~A}$ & 118.8 \\
\hline $\mathrm{C} 25-\mathrm{C} 26-\mathrm{H} 26 \mathrm{~A}$ & 119.1 & $\mathrm{C} 10-\mathrm{C} 9-\mathrm{H} 9 \mathrm{~A}$ & 118.8 \\
\hline $\mathrm{C} 27-\mathrm{C} 28-\mathrm{C} 19$ & $125.2(6)$ & $\mathrm{C} 9-\mathrm{C} 10-\mathrm{C} 1$ & $126.1(6)$ \\
\hline $\mathrm{C} 27-\mathrm{C} 28-\mathrm{C} 23$ & $119.5(6)$ & $\mathrm{C} 9-\mathrm{C} 10-\mathrm{C} 5$ & $117.8(5)$ \\
\hline $\mathrm{C} 19-\mathrm{C} 28-\mathrm{C} 23$ & $115.3(6)$ & $\mathrm{C} 1-\mathrm{C} 10-\mathrm{C} 5$ & $116.1(6)$ \\
\hline $\mathrm{O} 3-\mathrm{C} 29-\mathrm{N} 3$ & $120.2(5)$ & $\mathrm{O} 2-\mathrm{C} 11-\mathrm{N} 1$ & $121.6(5)$ \\
\hline $\mathrm{O} 3-\mathrm{C} 29-\mathrm{C} 22$ & $123.2(5)$ & $\mathrm{O} 2-\mathrm{C} 11-\mathrm{C} 6$ & $122.4(5)$ \\
\hline $\mathrm{N} 3-\mathrm{C} 29-\mathrm{C} 22$ & $116.6(5)$ & $\mathrm{N} 1-\mathrm{C} 11-\mathrm{C} 6$ & $116.0(5)$ \\
\hline $\mathrm{O} 4-\mathrm{C} 30-\mathrm{N} 3$ & $120.6(5)$ & $\mathrm{O} 1-\mathrm{C} 12-\mathrm{N} 1$ & $119.9(5)$ \\
\hline $\mathrm{O} 4-\mathrm{C} 30-\mathrm{C} 24$ & $123.1(5)$ & $\mathrm{O} 1-\mathrm{C} 12-\mathrm{C} 4$ & $124.3(5)$ \\
\hline $\mathrm{N} 3-\mathrm{C} 30-\mathrm{C} 24$ & $116.3(4)$ & $\mathrm{N} 1-\mathrm{C} 12-\mathrm{C} 4$ & $115.8(5)$ \\
\hline $\mathrm{C} 32-\mathrm{C} 31-\mathrm{N} 4$ & $111.0(5)$ & $\mathrm{C} 14-\mathrm{C} 13-\mathrm{N} 2$ & $109.8(5)$ \\
\hline $\mathrm{C} 32-\mathrm{C} 31-\mathrm{H} 31 \mathrm{~A}$ & 109.4 & $\mathrm{C} 14-\mathrm{C} 13-\mathrm{H} 13 \mathrm{~A}$ & 109.7 \\
\hline $\mathrm{N} 4-\mathrm{C} 31-\mathrm{H} 31 \mathrm{~A}$ & 109.4 & $\mathrm{~N} 2-\mathrm{C} 13-\mathrm{H} 13 \mathrm{~A}$ & 109.7 \\
\hline $\mathrm{C} 32-\mathrm{C} 31-\mathrm{H} 31 \mathrm{~B}$ & 109.4 & $\mathrm{C} 14-\mathrm{C} 13-\mathrm{H} 13 \mathrm{~B}$ & 109.7 \\
\hline $\mathrm{N} 4-\mathrm{C} 31-\mathrm{H} 31 \mathrm{~B}$ & 109.4 & $\mathrm{~N} 2-\mathrm{C} 13-\mathrm{H} 13 \mathrm{~B}$ & 109.7 \\
\hline $\mathrm{H} 31 \mathrm{~A}-\mathrm{C} 31-\mathrm{H} 31 \mathrm{~B}$ & 108.0 & $\mathrm{H} 13 \mathrm{~A}-\mathrm{C} 13-\mathrm{H} 13 \mathrm{~B}$ & 108.2 \\
\hline $\mathrm{C} 33-\mathrm{C} 32-\mathrm{C} 31$ & $179.0(7)$ & $\mathrm{C} 15-\mathrm{C} 14-\mathrm{C} 13$ & $177.1(9)$ \\
\hline $\mathrm{C} 32-\mathrm{C} 33-\mathrm{H} 33$ & 180.0 & $\mathrm{C} 14-\mathrm{C} 15-\mathrm{H} 15$ & 180.0 \\
\hline $\mathrm{C} 35-\mathrm{C} 34-\mathrm{N} 4$ & $112.8(5)$ & $\mathrm{C} 17-\mathrm{C} 16-\mathrm{N} 2$ & $110.5(5)$ \\
\hline $\mathrm{C} 35-\mathrm{C} 34-\mathrm{H} 34 \mathrm{~A}$ & 109.0 & $\mathrm{C} 17-\mathrm{C} 16-\mathrm{H} 16 \mathrm{~A}$ & 109.5 \\
\hline
\end{tabular}




\begin{tabular}{|c|c|c|c|}
\hline $\mathrm{N} 4-\mathrm{C} 34-\mathrm{H} 34 \mathrm{~A}$ & 109.0 & $\mathrm{~N} 2-\mathrm{C} 16-\mathrm{H} 16 \mathrm{~A}$ & 109.5 \\
\hline $\mathrm{C} 35-\mathrm{C} 34-\mathrm{H} 34 \mathrm{~B}$ & 109.0 & $\mathrm{C} 17-\mathrm{C} 16-\mathrm{H} 16 \mathrm{~B}$ & 109.5 \\
\hline $\mathrm{N} 4-\mathrm{C} 34-\mathrm{H} 34 \mathrm{~B}$ & 109.0 & $\mathrm{~N} 2-\mathrm{C} 16-\mathrm{H} 16 \mathrm{~B}$ & 109.5 \\
\hline $\mathrm{H} 34 \mathrm{~A}-\mathrm{C} 34-\mathrm{H} 34 \mathrm{~B}$ & 107.8 & $\mathrm{H} 16 \mathrm{~A}-\mathrm{C} 16-\mathrm{H} 16 \mathrm{~B}$ & 108.1 \\
\hline $\mathrm{C} 36-\mathrm{C} 35-\mathrm{C} 34$ & $178.3(7)$ & $\mathrm{C} 18-\mathrm{C} 17-\mathrm{C} 16$ & $178.6(7)$ \\
\hline $\mathrm{C} 35-\mathrm{C} 36-\mathrm{H} 36$ & 180.0 & $\mathrm{C} 17-\mathrm{C} 18-\mathrm{H} 18$ & 180.0 \\
\hline $\mathrm{C} 29-\mathrm{N} 3-\mathrm{N} 4-\mathrm{C} 34$ & $62.9(6)$ & $\mathrm{C} 12-\mathrm{N} 1-\mathrm{N} 2-\mathrm{C} 16$ & $58.3(6)$ \\
\hline $\mathrm{C} 30-\mathrm{N} 3-\mathrm{N} 4-\mathrm{C} 34$ & $-116.9(5)$ & $\mathrm{C} 11-\mathrm{N} 1-\mathrm{N} 2-\mathrm{C} 16$ & $-121.7(5)$ \\
\hline $\mathrm{C} 29-\mathrm{N} 3-\mathrm{N} 4-\mathrm{C} 31$ & $-66.0(6)$ & $\mathrm{C} 12-\mathrm{N} 1-\mathrm{N} 2-\mathrm{C} 13$ & $-72.5(6)$ \\
\hline $\mathrm{C} 30-\mathrm{N} 3-\mathrm{N} 4-\mathrm{C} 31$ & $114.2(5)$ & $\mathrm{C} 11-\mathrm{N} 1-\mathrm{N} 2-\mathrm{C} 13$ & $107.6(5)$ \\
\hline $\mathrm{C} 28-\mathrm{C} 19-\mathrm{C} 20-\mathrm{C} 21$ & $-1.4(10)$ & $\mathrm{C} 10-\mathrm{C} 1-\mathrm{C} 2-\mathrm{C} 3$ & $0.8(9)$ \\
\hline $\mathrm{Br} 2-\mathrm{C} 19-\mathrm{C} 20-\mathrm{C} 21$ & $178.6(5)$ & $\mathrm{Br} 1-\mathrm{C} 1-\mathrm{C} 2-\mathrm{C} 3$ & $-179.7(4)$ \\
\hline $\mathrm{C} 19-\mathrm{C} 20-\mathrm{C} 21-\mathrm{C} 22$ & $1.2(10)$ & $\mathrm{C} 1-\mathrm{C} 2-\mathrm{C} 3-\mathrm{C} 4$ & $-1.4(9)$ \\
\hline $\mathrm{C} 20-\mathrm{C} 21-\mathrm{C} 22-\mathrm{C} 23$ & $-1.3(9)$ & $\mathrm{C} 2-\mathrm{C} 3-\mathrm{C} 4-\mathrm{C} 5$ & $0.3(8)$ \\
\hline $\mathrm{C} 20-\mathrm{C} 21-\mathrm{C} 22-\mathrm{C} 29$ & $-178.4(5)$ & $\mathrm{C} 2-\mathrm{C} 3-\mathrm{C} 4-\mathrm{C} 12$ & $179.4(5)$ \\
\hline $\mathrm{C} 21-\mathrm{C} 22-\mathrm{C} 23-\mathrm{C} 24$ & $179.9(5)$ & $\mathrm{C} 3-\mathrm{C} 4-\mathrm{C} 5-\mathrm{C} 6$ & $-179.2(5)$ \\
\hline $\mathrm{C} 29-\mathrm{C} 22-\mathrm{C} 23-\mathrm{C} 24$ & $-3.1(8)$ & $\mathrm{C} 12-\mathrm{C} 4-\mathrm{C} 5-\mathrm{C} 6$ & $1.8(7)$ \\
\hline $\mathrm{C} 21-\mathrm{C} 22-\mathrm{C} 23-\mathrm{C} 28$ & $1.5(8)$ & $\mathrm{C} 3-\mathrm{C} 4-\mathrm{C} 5-\mathrm{C} 10$ & $1.3(8)$ \\
\hline $\mathrm{C} 29-\mathrm{C} 22-\mathrm{C} 23-\mathrm{C} 28$ & $178.5(5)$ & $\mathrm{C} 12-\mathrm{C} 4-\mathrm{C} 5-\mathrm{C} 10$ & $-177.8(5)$ \\
\hline $\mathrm{C} 22-\mathrm{C} 23-\mathrm{C} 24-\mathrm{C} 25$ & $-179.8(5)$ & $\mathrm{C} 4-\mathrm{C} 5-\mathrm{C} 6-\mathrm{C} 7$ & $-177.7(5)$ \\
\hline $\mathrm{C} 28-\mathrm{C} 23-\mathrm{C} 24-\mathrm{C} 25$ & $-1.4(7)$ & $\mathrm{C} 10-\mathrm{C} 5-\mathrm{C} 6-\mathrm{C} 7$ & $1.8(7)$ \\
\hline $\mathrm{C} 22-\mathrm{C} 23-\mathrm{C} 24-\mathrm{C} 30$ & $-0.1(7)$ & $\mathrm{C} 4-\mathrm{C} 5-\mathrm{C} 6-\mathrm{C} 11$ & $3.8(7)$ \\
\hline $\mathrm{C} 28-\mathrm{C} 23-\mathrm{C} 24-\mathrm{C} 30$ & $178.3(5)$ & $\mathrm{C} 10-\mathrm{C} 5-\mathrm{C} 6-\mathrm{C} 11$ & $-176.7(4)$ \\
\hline $\mathrm{C} 23-\mathrm{C} 24-\mathrm{C} 25-\mathrm{C} 26$ & $1.1(8)$ & $\mathrm{C} 5-\mathrm{C} 6-\mathrm{C} 7-\mathrm{C} 8$ & $-0.5(8)$ \\
\hline $\mathrm{C} 30-\mathrm{C} 24-\mathrm{C} 25-\mathrm{C} 26$ & $-178.5(5)$ & $\mathrm{C} 11-\mathrm{C} 6-\mathrm{C} 7-\mathrm{C} 8$ & $178.1(5)$ \\
\hline $\mathrm{C} 28-\mathrm{C} 27-\mathrm{C} 26-\mathrm{C} 25$ & $0.4(9)$ & $\mathrm{C} 6-\mathrm{C} 7-\mathrm{C} 8-\mathrm{C} 9$ & $-1.3(9)$ \\
\hline $\mathrm{C} 24-\mathrm{C} 25-\mathrm{C} 26-\mathrm{C} 27$ & $-0.6(9)$ & $\mathrm{C} 7-\mathrm{C} 8-\mathrm{C} 9-\mathrm{C} 10$ & $1.7(9)$ \\
\hline $\mathrm{C} 26-\mathrm{C} 27-\mathrm{C} 28-\mathrm{C} 19$ & $-179.5(6)$ & $\mathrm{C} 8-\mathrm{C} 9-\mathrm{C} 10-\mathrm{C} 1$ & $179.6(6)$ \\
\hline $\mathrm{C} 26-\mathrm{C} 27-\mathrm{C} 28-\mathrm{C} 23$ & $-0.6(9)$ & $\mathrm{C} 8-\mathrm{C} 9-\mathrm{C} 10-\mathrm{C} 5$ & $-0.3(8)$ \\
\hline $\mathrm{C} 20-\mathrm{C} 19-\mathrm{C} 28-\mathrm{C} 27$ & $-179.6(6)$ & $\mathrm{C} 2-\mathrm{C} 1-\mathrm{C} 10-\mathrm{C} 9$ & $-179.1(6)$ \\
\hline $\mathrm{Br} 2-\mathrm{C} 19-\mathrm{C} 28-\mathrm{C} 27$ & $0.5(8)$ & $\mathrm{Br} 1-\mathrm{C} 1-\mathrm{C} 10-\mathrm{C} 9$ & $1.5(8)$ \\
\hline $\mathrm{C} 20-\mathrm{C} 19-\mathrm{C} 28-\mathrm{C} 23$ & $1.5(9)$ & $\mathrm{C} 2-\mathrm{C} 1-\mathrm{C} 10-\mathrm{C} 5$ & $0.8(8)$ \\
\hline $\mathrm{Br} 2-\mathrm{C} 19-\mathrm{C} 28-\mathrm{C} 23$ & $-178.4(4)$ & $\mathrm{Br} 1-\mathrm{C} 1-\mathrm{C} 10-\mathrm{C} 5$ & $-178.7(4)$ \\
\hline $\mathrm{C} 24-\mathrm{C} 23-\mathrm{C} 28-\mathrm{C} 27$ & $1.1(8)$ & $\mathrm{C} 6-\mathrm{C} 5-\mathrm{C} 10-\mathrm{C} 9$ & $-1.5(7)$ \\
\hline $\mathrm{C} 22-\mathrm{C} 23-\mathrm{C} 28-\mathrm{C} 27$ & $179.5(5)$ & $\mathrm{C} 4-\mathrm{C} 5-\mathrm{C} 10-\mathrm{C} 9$ & $178.1(5)$ \\
\hline $\mathrm{C} 24-\mathrm{C} 23-\mathrm{C} 28-\mathrm{C} 19$ & $-179.9(5)$ & $\mathrm{C} 6-\mathrm{C} 5-\mathrm{C} 10-\mathrm{C} 1$ & $178.7(5)$ \\
\hline $\mathrm{C} 22-\mathrm{C} 23-\mathrm{C} 28-\mathrm{C} 19$ & $-1.5(7)$ & $\mathrm{C} 4-\mathrm{C} 5-\mathrm{C} 10-\mathrm{C} 1$ & $-1.8(7)$ \\
\hline $\mathrm{N} 4-\mathrm{N} 3-\mathrm{C} 29-\mathrm{O} 3$ & $1.8(8)$ & $\mathrm{C} 12-\mathrm{N} 1-\mathrm{C} 11-\mathrm{O} 2$ & $174.4(5)$ \\
\hline $\mathrm{C} 30-\mathrm{N} 3-\mathrm{C} 29-\mathrm{O} 3$ & $-178.4(5)$ & $\mathrm{N} 2-\mathrm{N} 1-\mathrm{C} 11-\mathrm{O} 2$ & $-5.6(7)$ \\
\hline $\mathrm{N} 4-\mathrm{N} 3-\mathrm{C} 29-\mathrm{C} 22$ & $-177.6(4)$ & $\mathrm{C} 12-\mathrm{N} 1-\mathrm{C} 11-\mathrm{C} 6$ & $-4.6(7)$ \\
\hline $\mathrm{C} 30-\mathrm{N} 3-\mathrm{C} 29-\mathrm{C} 22$ & $2.2(7)$ & $\mathrm{N} 2-\mathrm{N} 1-\mathrm{C} 11-\mathrm{C} 6$ & $175.3(4)$ \\
\hline $\mathrm{C} 21-\mathrm{C} 22-\mathrm{C} 29-\mathrm{O} 3$ & $-0.2(8)$ & $\mathrm{C} 7-\mathrm{C} 6-\mathrm{C} 11-\mathrm{O} 2$ & $-0.2(8)$ \\
\hline $\mathrm{C} 23-\mathrm{C} 22-\mathrm{C} 29-\mathrm{O} 3$ & $-177.3(5)$ & $\mathrm{C} 5-\mathrm{C} 6-\mathrm{C} 11-\mathrm{O} 2$ & $178.4(5)$ \\
\hline $\mathrm{C} 21-\mathrm{C} 22-\mathrm{C} 29-\mathrm{N} 3$ & $179.2(5)$ & $\mathrm{C} 7-\mathrm{C} 6-\mathrm{C} 11-\mathrm{N} 1$ & $178.9(5)$ \\
\hline $\mathrm{C} 23-\mathrm{C} 22-\mathrm{C} 29-\mathrm{N} 3$ & $2.1(7)$ & $\mathrm{C} 5-\mathrm{C} 6-\mathrm{C} 11-\mathrm{N} 1$ & $-2.5(7)$ \\
\hline $\mathrm{C} 29-\mathrm{N} 3-\mathrm{C} 30-\mathrm{O} 4$ & $175.9(5)$ & $\mathrm{C} 11-\mathrm{N} 1-\mathrm{C} 12-\mathrm{O} 1$ & $-170.7(5)$ \\
\hline $\mathrm{N} 4-\mathrm{N} 3-\mathrm{C} 30-\mathrm{O} 4$ & $-4.4(7)$ & $\mathrm{N} 2-\mathrm{N} 1-\mathrm{C} 12-\mathrm{O} 1$ & $9.4(8)$ \\
\hline
\end{tabular}




$\begin{array}{ll}\mathrm{C} 29-\mathrm{N} 3-\mathrm{C} 30-\mathrm{C} 24 & -5.1(7) \\ \mathrm{N} 4-\mathrm{N} 3-\mathrm{C} 30-\mathrm{C} 24 & 174.6(4) \\ \mathrm{C} 25-\mathrm{C} 24-\mathrm{C} 30-\mathrm{O} 4 & 2.6(8) \\ \mathrm{C} 23-\mathrm{C} 24-\mathrm{C} 30-\mathrm{O} 4 & -177.0(5) \\ \mathrm{C} 25-\mathrm{C} 24-\mathrm{C} 30-\mathrm{N} 3 & -176.3(5) \\ \mathrm{C} 23-\mathrm{C} 24-\mathrm{C} 30-\mathrm{N} 3 & 4.0(7) \\ \mathrm{N} 3-\mathrm{N} 4-\mathrm{C} 31-\mathrm{C} 32 & -51.7(6) \\ \mathrm{C} 34-\mathrm{N} 4-\mathrm{C} 31-\mathrm{C} 32 & 178.6(5) \\ \mathrm{N} 4-\mathrm{C} 31-\mathrm{C} 32-\mathrm{C} 33 & 176(100) \\ \mathrm{N} 3-\mathrm{N} 4-\mathrm{C} 34-\mathrm{C} 35 & 51.8(6) \\ \mathrm{C} 31-\mathrm{N} 4-\mathrm{C} 34-\mathrm{C} 35 & -178.3(5) \\ \mathrm{N} 4-\mathrm{C} 34-\mathrm{C} 35-\mathrm{C} 36 & -157(25)\end{array}$

$\begin{array}{ll}\mathrm{C} 11-\mathrm{N} 1-\mathrm{C} 12-\mathrm{C} 4 & 9.8(7) \\ \mathrm{N} 2-\mathrm{N} 1-\mathrm{C} 12-\mathrm{C} 4 & -170.1(4) \\ \mathrm{C} 3-\mathrm{C} 4-\mathrm{C} 12-\mathrm{O} 1 & -6.8(9) \\ \mathrm{C} 5-\mathrm{C} 4-\mathrm{C} 12-\mathrm{O} 1 & 172.3(5) \\ \mathrm{C} 3-\mathrm{C} 4-\mathrm{C} 12-\mathrm{N} 1 & 172.8(5) \\ \mathrm{C} 5-\mathrm{C} 4-\mathrm{C} 12-\mathrm{N} 1 & -8.2(7) \\ \mathrm{N} 1-\mathrm{N} 2-\mathrm{C} 13-\mathrm{C} 14 & -171.5(5) \\ \mathrm{C} 16-\mathrm{N} 2-\mathrm{C} 13-\mathrm{C} 14 & 59.6(7) \\ \mathrm{N} 2-\mathrm{C} 13-\mathrm{C} 14-\mathrm{C} 15 & -99(15) \\ \mathrm{N} 1-\mathrm{N} 2-\mathrm{C} 16-\mathrm{C} 17 & 55.8(6) \\ \mathrm{C} 13-\mathrm{N} 2-\mathrm{C} 16-\mathrm{C} 17 & -175.6(5) \\ \mathrm{N} 2-\mathrm{C} 16-\mathrm{C} 17-\mathrm{C} 18 & -52(32)\end{array}$

